\title{
Modal processing for acoustic communications in shallow water experiment
}

\author{
Andrey K. Morozov, James C. Preisig, and Joseph Papp \\ Department of Applied Ocean Physics and Engineering, Woods Hole Oceanographic Institution, Woods Hole, \\ Massachusetts 02543 \\ amorozov@whoi.edu,jpreisig@whoi.edu,jpapp@whoi.edu
}

\begin{abstract}
Acoustical array data from the Shallow Water Acoustics experiment was processed to show the feasibility of broadband mode decomposition as a preprocessing method to reduce the effective channel delay spread and concentrate received signal energy in a small number of independent channels. The data were collected by a vertical array designed at the Woods Hole Oceanographic Institution. Phase-shift Keying (PSK) $m$-sequence modulated signals with different carrier frequencies were transmitted at a distance $19.2 \mathrm{~km}$ from the array. Even during a strong internal waves activity a low bit error rate was achieved.

(C) 2008 Acoustical Society of America

PACS numbers: 43.30.Bp [WC]

Date Received: January 22, 2008 Date Accepted: April 4, 2008
\end{abstract}

\section{Introduction}

Two principle factors that limit the performance of adaptive coherent underwater acoustic communications systems are the ability to adapt the system rapidly enough to keep up with environmental fluctuations or platform motion and a lack of adequate signal energy. The former limitation arises in the context of adaptive equalizers that must estimate the channel impulse response and calculate filter coefficients as the environmental conditions change. The rate of adaptation is limited by the number of degrees of freedom (e.g., the number of taps in the channel impulse response or the equalizer filters) that must be adjusted.

A natural framework for addressing these limiting factors in low frequency acoustic communications is that of mode propagation. In the cases where the receiver has access to an array with enough sensors and aperture to resolve the propagating modes [e.g., a fixed base station that receives signals from autonomous underwater vehicles (AUVs)], wideband modal filtering as an initial processing stage can reduce the number of degrees of freedom (e.g., reduce the effective delay spread of the channel impulse response) that need to be adjusted in later adaptive stages and provides a method of coherently combining all of the received propagating energy in the water column. The former advantage is realized for two reasons. The first is that the number of propagating modes and thus the number of modes containing the significant energy is often less than the number of sensors. Therefore, a problem of working on the outputs of $N$ sensors can be reduced to the problem of operating on the wideband modal time series of $M$ modes where $M<N$. The second reason is that the temporal dispersion of the energy propagating in a single mode is always less than or equal to the total temporal dispersion of the channel. The magnitude of the inequality is dependent on the amount of modal coupling in the propagation channel as well as bandwidth of the transmitted signal. The ability to coherently combine all of the propagating energy in the water column is inherent in the use of modal filtering over all of the propagating modes.

The objectives of this research are to process and analyze the data collected during the Shallow Water acoustics experiment 2006 (SW06) to demonstrate the feasibility of broadband mode decomposition as a preprocessing method to reduce the effective channel delay spread and concentrate received signal energy in a small number of independent channels. 


\section{Experiment conditions}

The processed data were collected by a 48-element array called SHARK, designed at the Woods Hole Oceanographic Institution (Newhall et al., 2007). The array system included a 16 channel vertical array (VLA) and a 32 channel horizontal array. The system was deployed in $78 \mathrm{~m}$ of water, which allowed 14 of the 16 vertical array channels to span the water column from about $78 \mathrm{~m}$ to $12 \mathrm{~m}$ depth. The hydrophone depths (not equidistant) are: 13.5 (top), 17.25, 21, 24.75, $28.5,32.25,36,39.75,43.5,47.25,54.75,62.25,69.75,77.25,77.75$, and $77.75 \mathrm{~m}$.

For broadband mode processing, the first 14 hydrophones were used. The following signals, emitted from the Miami sound machine (MSM) sound source (Newhall et al., 2007) along the bottom slope from the distance $19.2 \mathrm{~km}$, have been processed: Periodically repeated 63 digit $M$-sequence signal with the carrier frequency $101.7 \mathrm{~Hz} ; 127$ digit $M$-sequence signal with the frequency $203.4 \mathrm{~Hz} ; 511$ digit $M$ sequence with frequency $813.8 \mathrm{~Hz}$. The signal was phase modulated with $180^{\circ}$ phase shift, four carrier cycles per digit, $40 M$-sequence periods were processed. The phase shifts take place at zero crossings.

The sound velocity was measured by ten sensors attached to the VLA at the depths: 13 , $14.8,18.5,22.3,26,33.6,41,56,71$, and $78.3 \mathrm{~m}$. The sound velocity was measured every $30 \mathrm{~s}$. The mode processing used an averaged value of the sound velocity profile for the time interval covering the signal length.

\section{Broadband mode filtering}

A number of papers have been dedicated to shallow water acoustic mode problems. Acoustic normal mode filtering and mode fluctuations in shallow water experiments were considered in the paper by Headrick et al. (2000). The comparison of different approaches to mode coefficients estimation was conducted in the papers by Buck et al. (1998), Wage et al. (2003), and Wage et al. (2005). In accordance to referenced sources the pseudo-inverse transformation is a very reasonable method for mode coefficient estimation when signal to noise ratio is much larger than one. The first results of mode fluctuations in SW06 were considered by Duda et al. (2007), and Collis (2007).

The broadband mode filtering was implemented as follows. The input signal for each hydrophone was sampled and divided into sections. Each section for each hydrophone was zero padded and then Fourier transformed. For each frequency, the Moore-Penrose pseudo inverse of the sampled mode shape matrix was calculated. The pseudo-inverse matrix computation was based on singular value decomposition with large singular values. At each frequency, this inverse was used to perform the modal filtering and the resulting filtered signals were transformed to low frequencies and then inverse Fourier transformed to obtain the modal time series for each mode. The processed sections then were down sampled and combined by overlap-add method. The mode filtering was followed by a correlation with the initial $M$-sequence phasemanipulated signal, and signal demodulation analysis. Another approach based on the low-pass equivalent method (base-band mode filtering) was tested and gave the equivalent result with less computation.

The calculation of the mode shapes used for the mode filtering used data from sound velocity sensors averaged over signal transmission interval. Bottom sound velocity, density, and attenuation were taken from from the paper by Yong-Min Jiang et al. (2007). A standard Kraken algorithm was applied for each frequency in the signal bandwidth to calculate frequency dependent acoustic modes and mode wave numbers. Each $m$-sequence signal has four carrier periods in one bit and its bandwidth was $2 \Delta f=0.25 f_{0}$, where $f_{0}$ is the carrier frequency. The frequency dependent mode functions were calculated for the whole signal frequency bandwidth.

\section{Correlation of mode filter output with the $M$-sequence signal}

The correlation of the signal after the mode filter with the cyclically time-shifted replica of transmitted $M$-sequence phase manipulated signal shows the delay spread of acoustic energy. Correlation of the received signal with the reference signal with a shifted carrier frequency shows that there was no relevant frequency Doppler shift of the received signals. The results for 


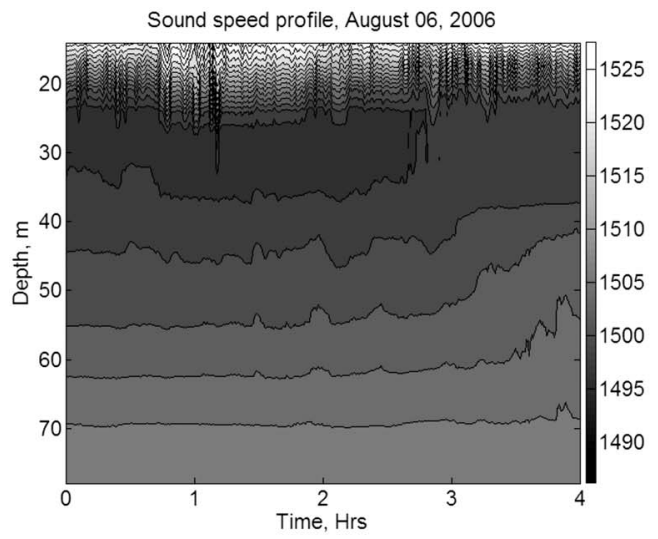

A

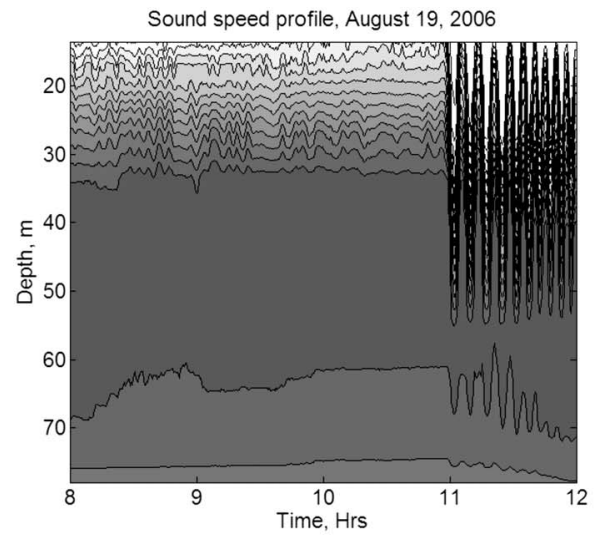

B

Fig. 1. Sound speed fluctuations.

two different cases are presented below. The first case is "typical." It is a usual internal wave activity, which was observed during most of the SW06 experiment. The second case has "extremely strong" internal wave activity, which leads to strong fluctuations of channel parameters. The last case is not typical, it has been observed only once during the SW06 experiment. These two examples are presented in Fig. 1: ordinary internal wave fluctuations (A) and extremely strong internal wave solitons (B). A tidal wave presents in Fig. 1(a). The correlation functions for these two cases for the carrier $203 \mathrm{~Hz}$ are shown in Fig. 2. The constellations of complex envelopes at the output of the mode filters for mode 2 (case A) and mode 1 (case B) are presented in Fig. 3. Signal constellations are not bad and high quality data reception is probable.

\section{Data processing results}

The good shape of the signal constellations diagram at the output of the mode filter suggests that good results can be expected from data recovery by a short length Viterbi decoder with a simple channel parameters estimator. A joint channel estimation and data recovery algorithm identical to Morozov et al. (2006) was applied for quality of data reception demonstration.

The discrete form of the channel at the output of a mode filter is

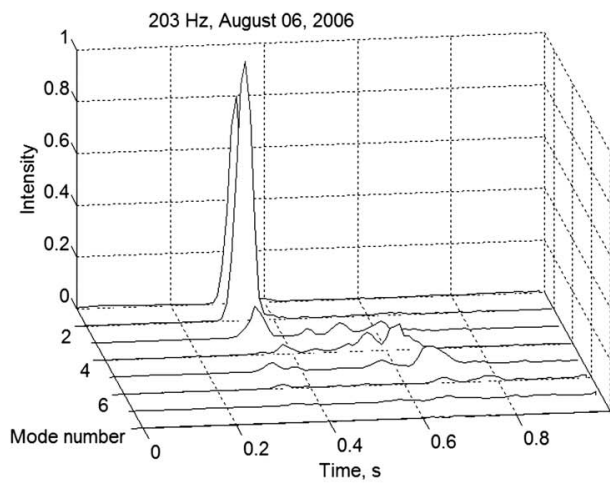

A

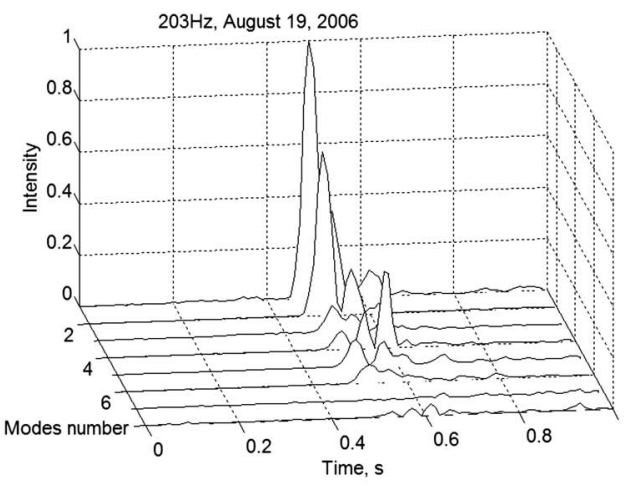

B

Fig. 2. Mode-time correlations. 

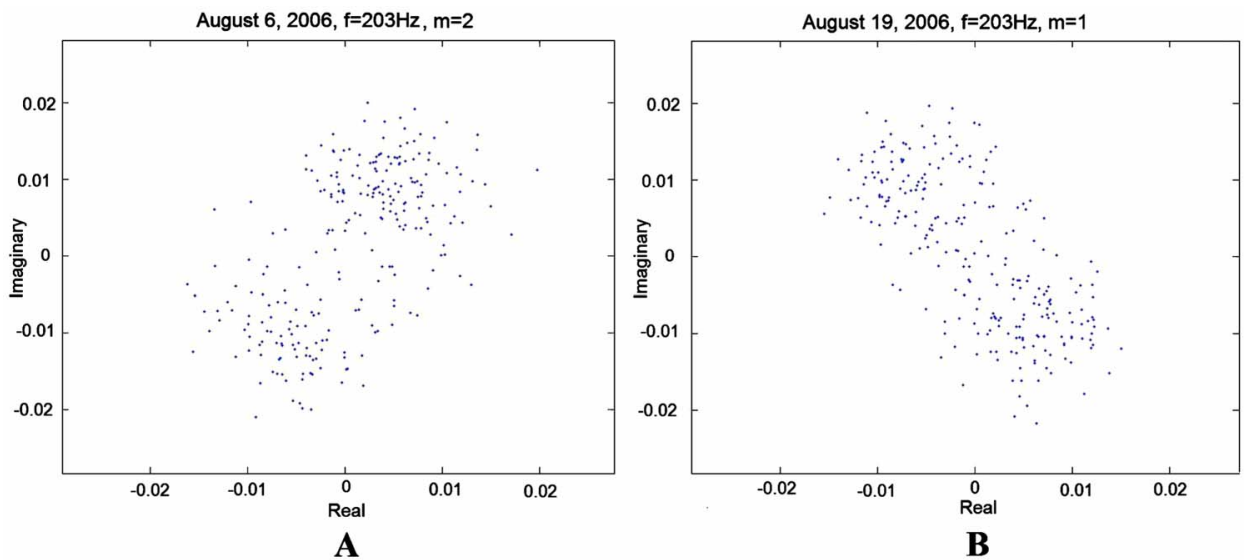

Fig. 3. (Color online) Signal complex envelop constellations at the output of mode filters.

$$
y_{l}=\sum_{j=1}^{N} h_{l j} k_{l-j}+n_{l},
$$

where $n_{l}$ is the white Gaussian noise and $h_{j l}$ is the low-frequency equivalent of time-variable channel pulse response; $k_{j}= \pm 1$ is the modulating parameter.

The simplified equations for the maximum-likelihood metrics $w_{l}$ are

$$
w_{l}=w_{l-1}-\left|d_{l}\right|^{2} ; \quad \hat{h}_{l m}=\hat{h}_{l-1, m}-a d_{l} k_{l-m} ; \quad d_{l}=y_{l}-\sum_{m=0}^{N} \hat{h}_{l m} k_{l-m},
$$

where $\hat{h}_{l m}$ are least mean squares (LMS) estimates of the channel impulse response obtained in Equ. (2); $a=0.01$ is the step size of the estimation algorithm. The algorithm operates by iterations for each sample with the rate of two samples per pulse. The algorithm calculates trellis metrics in accordance with Eq. 2 and includes an LMS adaptive channel estimator for each sequence of data symbols (for each branch of the trellis).

A receiver estimated mode-time intensity distribution and used the most energetic modes for demodulation. For case A, the data processing gave a bit error rate (BER) of 0.2 , when the signal from the first mode filter was received, the $\mathrm{BER}=0$ for the second mode and 0.2 for the third mode. For case B and the first mode, BER was $0.2, \mathrm{BER}=0$ for the second mode, and 0.49 for the third mode. In both cases the second mode filtering gave the best reception quality with no errors.

These results demonstrate that even in a very complicated environment with strong internal waves solitons the data can be transmitted over the distance $19.2 \mathrm{~km}$ without errors. Acoustical energy was concentrated in a small number of the first acoustical modes. Note that the sound pressure level of the transmitter was limited to approximately $186 \mathrm{~dB}$.

\section{Conclusion}

Broadband mode filtering allows one to decompose the received signal into a number of independent signals with a reduced channel delay spread. The data processing shows that even in a very complicated environment with strong internal wave solitons the acoustical energy is concentrated in a small number of the first acoustical modes. A receiver can estimate mode-time intensity distribution and use a signal from a more intensive mode (or a few of them) for demodulation. A very high quality data transmission can be achieved for a range of approximately $20 \mathrm{~km}$. 


\section{Acknowledgments}

The authors express sincere thanks to Dr. James Lynch and Arthur Newhall for SW06 experimental data. The authors deeply thank Dr. Timothy Duda, Dr. Jon Collis, Dr. Ying Tsong Lin for the help in data processing, Keith Von Der Heydt for the SHARK acoustic array design, and Dr. Harry DeFerrari for the MSM underwater acoustic sound source. The research was supported by ONR.

\section{References and links}

Buck, J. R., Preisig, J. C., Wage, K. E., (1998). "A unified framework for mode filtering and the maximum a posteriori mode filter," J. Acoust. Soc. Am. 103, 1813-1824.

Collis, J. M., Duda, T. F., Lynch, J. F., and DeFerrari, H. A., (2008). "Observed limiting cases of horizontal field cohrerence and array performance in a time-varying internal wave- field," J. Acoust. Soc. Am. 124,

EL97-EL103.

Duda, T. F., and Collis, J. M., (2007) "Acoustic field coherence in four-dimensionally variable shallow water environments: Estimation using co-located horizontal and vertical line arrays," Proceedings of the conference "Underwater Acoustic Measurements, Technologies and Results," Crete, Greece.

Headrick, R. H., Lynch, J. F., Kemp, J. N., Newhall, A. E., von der Heydt, K., Apel, J., Badey, M., Chiu, C.-S., Finette, S., Orr, M., Pasewark, B., Turdot, A., Wolf, S., and Tielbuerger, D., (2000). “Acoustic normal mode fluctuation statistics in 1995 SWARM internal wave scattering experiment,” J. Acoust. Soc. Am. 107, 201-220.

Morozov, A. K., and Preisig, J. C., (2006). "Underwater acoustic communications with multi-carrier modulation." Proceedings of Oceans 2006.

Newhall, A. E., Duda, T. F., Von der Heydt, K., Irish, J. D., Kemp, J. N., Lerner, S. A., Liberatore, S. P., Lin, Y. T., Lynch, J. F., Maffei, A. R., Morozov, A. K., Shmelev, A. A., Sellers, C. J., and Witzell, W., (2007). "Acoustic and oceanographic observations and configuration information for the WHOI moorings from the SW06 experiment," Applied Ocean Physics and Engineering (AOP\&E), WHOI Technical Reports.

Wage, K. E., Baggeroer, A. B., and Preisig, J. C., (2003). "Modal analysis of broadband acoustic receptions at 3515-km range in North Pacific using short-time Fourier techniques," J. Acoust. Soc. Am. 113, 801-817.

Wage, K. E., Dzieciuch, M. A., Worcester, P. F., Howe, B. M., and Mercer, J. A., (2005). "Mode coherence at megameter ranges in North Pacific Ocean,” J. Acoust. Soc. Am. 117, 1565-1581.

Yong-Min, Jiang, Chapman, N. Ross, and Badiey, Mohsen, (2007). "Quantifying the uncertainty of geoacoustic parameter estimates for the New Jersey shelf by inverting air gun data," J. Acoust. Soc. Am. 121, 4, 1879-1894. 